\section{(1)}

CrossMark

\title{
Excess mucus viscosity and airway dehydration impact COPD airway clearance
}

\author{
Vivian Y. Lin ${ }^{1}$, Niroop Kaza ${ }^{1}$, Susan E. Birket ${ }^{1,2}$, Harrison Kim $\mathbb{1}^{3}$, Lloyd \\ J. Edwards ${ }^{4}$, Jennifer LaFontaine ${ }^{2}$, Linbo Liu ${ }^{5}$, Marina Mazur ${ }^{2}$, Stephen \\ A. Byzek ${ }^{2}$, Justin Hanes ${ }^{6}$, Guillermo J. Tearney ${ }^{7}$, S. Vamsee Raju ${ }^{1,2}$ and \\ Steven M. Rowe (1) ${ }^{1,2}$
}

Affiliations: ${ }^{1}$ Dept of Medicine, University of Alabama at Birmingham, Birmingham, AL, USA. ${ }^{2}$ Cystic Fibrosis Research Center, University of Alabama at Birmingham, Birmingham, AL, USA. ${ }^{3}$ Dept of Radiology, University of Alabama at Birmingham, Birmingham, AL, USA. ${ }^{4}$ Dept of Biostatistics, University of Alabama at Birmingham, Birmingham, AL, USA. ${ }^{5}$ School of Electrical \& Electronic Engineering and School of Chemical \& Biomedical Engineering, Nanyang Technological University, Singapore. ${ }^{6}$ The Center for Nanomedicine at Wilmer Eye Institute, Johns Hopkins University, Baltimore, MD, USA. 'Wellman Center for Photomedicine, Massachusetts General Hospital, Boston, MA, USA.

Correspondence: Steven M. Rowe, 1918 University Blvd, MCLM 702, Birmingham, AL 35294, USA. E-mail: srroweduabmc.edu

@ERSpublications

Mucus abnormalities impact COPD airway clearance http://bit.ly/2VEhFjd

Cite this article as: Lin VY, Kaza N, Birket SE, et al. Excess mucus viscosity and airway dehydration impact COPD airway clearance. Eur Respir J 2020; 55: 1900419 [https://doi.org/10.1183/13993003.004192019].

ABSTRACT The mechanisms by which cigarette smoking impairs airway mucus clearance are not well understood. We recently established a ferret model of cigarette smoke-induced chronic obstructive pulmonary disease (COPD) exhibiting chronic bronchitis. We investigated the effects of cigarette smoke on mucociliary transport (MCT).

Adult ferrets were exposed to cigarette smoke for 6 months, with in vivo mucociliary clearance measured by technetium-labelled DTPA retention. Excised tracheae were imaged with micro-optical coherence tomography. Mucus changes in primary human airway epithelial cells and ex vivo ferret airways were assessed by histology and particle tracking microrheology. Linear mixed models for repeated measures identified key determinants of MCT.

Compared to air controls, cigarette smoke-exposed ferrets exhibited mucus hypersecretion, delayed mucociliary clearance $(-89.0 \%, \mathrm{p}<0.01)$ and impaired tracheal MCT $(-29.4 \%, \mathrm{p}<0.05)$. Cholinergic stimulus augmented airway surface liquid (ASL) depth $(5.8 \pm 0.3$ to $7.3 \pm 0.6 \mu \mathrm{m}, \mathrm{p}<0.0001)$ and restored MCT $\left(6.8 \pm 0.8\right.$ to $\left.12.9 \pm 1.2 \mathrm{~mm} \cdot \mathrm{min}^{-1}, \mathrm{p}<0.0001\right)$. Mixed model analysis controlling for covariates indicated smoking exposure, mucus hydration (ASL) and ciliary beat frequency were important predictors of MCT. Ferret mucus was hyperviscous following smoke exposure in vivo or in vitro, and contributed to diminished MCT. Primary cells from smokers with and without COPD recapitulated these findings, which persisted despite the absence of continued smoke exposure.

Cigarette smoke impairs MCT by inducing airway dehydration and increased mucus viscosity, and can be partially abrogated by cholinergic secretion of fluid secretion. These data elucidate the detrimental effects of cigarette smoke exposure on mucus clearance and suggest additional avenues for therapeutic intervention.

This article has an editorial commentary: https://doi.org/10.1183/13993003.02473-2019

This article has supplementary material available from erj.ersjournals.com

Received: 28 Feb 2019 | Accepted after revision: 9 Oct 2019

Copyright OERS 2020 


\section{Introduction}

Chronic obstructive pulmonary disease (COPD) is the third leading cause of death in the United States and is increasing in incidence worldwide [1]. Although the primary risk factor is cigarette smoking [2], pathogenesis continues after smoking cessation [3] and environmental exposures or genetics can cause COPD in nonsmokers $[2,4,5]$.

Individuals with chronic bronchitis exhibit mucus expectoration, airway inflammation [6] and delayed mucus clearance $[7,8]$. The underlying mechanisms are not yet elucidated, although mucus hypersecretion $[9,10]$, airway dehydration $[11,12]$ and rheological alterations [13] are postulated to cause muco-obstruction. Mucin hyperconcentration associated with disease severity might also underlie obstruction and exacerbations [14,15]. Understanding their contributions will facilitate targeting these pathways for novel interventions.

We recently developed a COPD ferret model [16], which offers several advantages over traditional rodent models. For instance, ferret airways better approximate human goblet cell and glandular distribution [17, 18], yielding a mucus phenotype that includes chronic bronchitis and risk of spontaneous respiratory exacerbations, features that smoke-exposed mice lack $[19,20]$. Coupling co-localised estimates of airway epithelial function [21] with mucus measurements, we can assess mucociliary transport (MCT) abnormalities and contributing physiological mechanisms not feasible in humans.

Here, we hypothesised that chronic cigarette smoke impairs the MCT apparatus by affecting mucus hydration and viscosity, and is important in slowing mucus clearance in COPD. To accomplish this, we assessed smoke-induced defects in the airway functional microanatomy, identifying factors that determine mucus transport in a faithful model of chronic bronchitis pathogenesis.

\section{Methods}

Study approval

The University of the Alabama at Birmingham institutional animal care and use committee reviewed and approved animal studies (IACUC-20232, -20073 and -20232), and the institutional review board approved human tissue use (IRB-160901001 and -101111015). All subjects provided written informed consent for use of cells and tissues.

\section{Cell culture}

Primary human bronchial epithelial (HBE) cells were isolated from tissue from normal (nonsmoker), healthy smoker (lacking lung disease) and COPD donors of varying sex, race and age, then cultured at the air-liquid interface until fully differentiated $[22,23]$.

\section{Smoke exposure}

Age- and sex-matched wild-type ferrets (Marshall BioResources, North Rose, NY, USA) were exposed to room air or nose-only smoke $\left(5 \mathrm{~h} \cdot \mathrm{day}^{-1}, 5\right.$ days $\cdot \mathrm{week}^{-1}, 6$ months) generated by automated systems as previously described [16].

\section{In vivo mucociliary clearance}

Anaesthetised ferrets were intubated $(2.5 \mathrm{~mm}$ intratracheal tube extending $12 \mathrm{~cm})$ and received aerosols of technetium-labelled DTPA (Tc-DPTA) (up to $50 \mathrm{mCi} \cdot \mathrm{kg}^{-1}$ body weight) generated by a raindrop nebuliser (Aeroneb; Aerogen, Galway, Ireland) in a safety hood that allows for the ventilation of radioactive materials. Ferrets inhaled the radiolabelled aerosols generated from a $1 \mathrm{~mL}$ dilution in saline over $5 \mathrm{~min}$. A $\gamma$-camera (Gamma Medica-Ideas, Northridge, CA, USA) was used to measure the clearance of Tc-DTPA from both lungs for the next $60 \mathrm{~min}$. Measures of time-dependent clearance were obtained from the entire lungs demarked with parallel computed tomography imaging. External radiolabelled markers were placed on the ferret to facilitate proper alignment under the $\gamma$-camera and to account for decay.

The rate of mucociliary clearance (MCC) was corrected for decay and expressed as percentage of radioactivity present in the initial baseline image after normalising for systemic absorption of DTPA. The measurement of MCC was based on modifications to a previously published method that showed that the elimination kinetics of indium-DTPA represent the combination of MCC and absorptive clearance [24]. Absorptive clearance of Tc-DTPA was measured by monitoring its uptake into arm muscles (biceps and triceps). MCC was calculated by subtracting absorptive clearance from total clearance.

\section{Micro-optical coherence tomography}

Micro-optical coherence tomography $(\mu \mathrm{OCT})$-recorded images of normal HBE cells and excised ferret trachea were analysed using ImageJ (National Institutes of Health, Bethesda, MD, USA) and MATLAB software (MathWorks, Natick, MA, USA) to assess airway epithelial function [21]. Tissues were 
maintained on gauze saturated in warmed DMEM (Gibco, Waltham, MA, USA) containing $1 \mu \mathrm{M}$ indomethacin (to inhibit prostaglandin signalling) [25] for images recorded immediately following excision. This was replaced with indomethacin and carbachol $(1 \mu \mathrm{M}$ each, $30 \mathrm{~min}$; Sigma-Aldrich, St Louis, MO, USA) to stimulate secretion for additional MCT measurements before mucus collection (supplementary figure S2).

\section{Mucus collection}

Mucus was collected from unstimulated HBE cells for immediate use. Following $\mu \mathrm{OCT}$ video acquisition, ferret trachea were plugged at the ends to facilitate accumulation, then treated with $10 \mu \mathrm{M}$ carbachol and $20 \mu \mathrm{M}$ phenylephrine $(2 \mathrm{~h}$; Sigma-Aldrich) to maximally stimulate secretion sufficient for subsequent assays (supplementary figure S2).

\section{Particle tracking microrheology}

Mucus samples containing fluorescent polyethylene glycol-polystyrene (500-nm or $1-\mu \mathrm{m})$ particles $(1: 10 \mathrm{v} /$ $\mathrm{v}$ dilution) were loaded underneath a coverslip $(10 \mu \mathrm{L})$ to assess Brownian motion of individual particles. Following 1-h incubation, samples were imaged using $\mu$ OCT or fluorescent microscopy (Nikon TE200; Nikon Instruments, Melville, NY, USA) as described previously [26].

\section{Mucus percentage solids by weight}

Aluminium foil pieces were tared on a UMX2 Ultra-microbalance (Mettler Toledo, Columbus, OH, USA) before weighing mucus samples $\left(5 \mu \mathrm{L} \mathrm{HBE}, 1-5 \mu \mathrm{L}\right.$ ferret). Samples were dried overnight $\left(80^{\circ} \mathrm{C}\right)$ for percentage nonvolatile solid calculation.

\section{Statistical analysis}

All quantitative measures were analysed using descriptive statistics and regression methods in GraphPad Prism (GraphPad Software, La Jolla, CA, USA), SPSS Statistics (IBM, Armonk, NY, USA) or SAS v9.4 (Cary, NC, USA). Data are presented as mean \pm SE unless otherwise specified, with p-values $<0.05$ considered statistically significant. Further details regarding methods and statistical analyses are given in the supplementary material.

\section{Results}

\section{Mucus clearance is reduced in a ferret model of COPD}

We recently developed a cigarette smoke-induced ferret model of COPD and chronic bronchitis [16] demonstrating goblet cell hyperplasia, chronic cough and episodic exacerbations reminiscent of human disease but not found in mouse models, providing an opportunity to identify physiological mechanisms and determine relationships to MCT apparatus abnormalities. We first measured in vivo MCC using Tc-DTPA retention (figure 1a) over time after subtracting the amount of label that eliminated via absorption; heat maps of MCT were developed to represent clearance rates. Following comparable deposition of the radiolabel in each exposure group (supplementary figure S1), quantification of these images showed reduced clearance in smoke-exposed ferrets in $60 \mathrm{~min}$ (2.8\% versus $25.4 \%$ air controls, $\mathrm{p}<0.01$ ) (figure $1 \mathrm{~b}$ ). The area under the curve for each group demonstrated significantly higher percentage retention after $60 \mathrm{~min}$ in smoke-exposed ferrets $(2923 \pm 86$ smoke versus $2543 \pm 75$ air control, $\mathrm{p}<0.01)$, indicating a markedly slower MCC rate compared to air controls (figure 1c). In addition, a diminished rate of MCC was evident when the remaining amount of Tc-DTPA after $60 \mathrm{~min}$ was measured (figure 1d), despite higher clearance of the label via absorption in smoke-exposed ferrets.

\section{Airway epithelial dysfunction impairs mucus transport in COPD}

Using $\mu \mathrm{OCT}$ analysis of excised ferret trachea (figure $2 \mathrm{a}$ and $\mathrm{b}$, supplementary figure S2), we next sought to characterise the physiological defects within the MCT apparatus and their connections with potentially abnormal mucus. When quantified as single point estimates for individual ferrets, there was a $29.4 \%$ reduction in MCT (figure 2c) in COPD ferrets $\left(6.8 \pm 0.8\right.$ versus $9.6 \pm 1.1 \mathrm{~mm} \cdot \mathrm{min}^{-1}$ air controls, $\left.\mathrm{p}<0.05\right)$, parallelling the reduction seen in vivo. A linear mixed model for repeated measures within each ferret and adjusting for two measures at baseline, cohort and sex, confirmed the deleterious effects of smoking on MCT (32\% reduction, $\mathrm{p}=0.016, \mathrm{R}^{2}=0.368$ ). Seeking potential explanations, we note that estimates by outcome were variable: both airway surface liquid (ASL) (figure 2d) and periciliary layer (PCL) (figure 2e), markers of airway hydration, exhibited small non-statistically significant reductions in cigarette smoke-exposed ferrets (by $12 \%$ and $2.9 \%$, respectively), whereas there was no meaningful difference in ciliary beat frequency (CBF) (figure $2 \mathrm{f}$ ).

To better account for the microenvironment and their interrelationships (factors known to influence each other $[21,27,28]$ ), we developed a statistical model that included all known covariates of MCT that were 


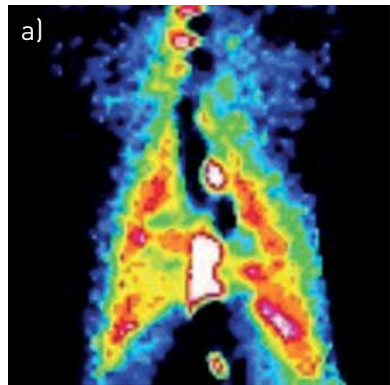

Control

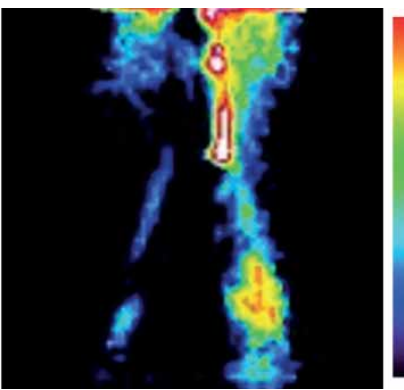

Smoke

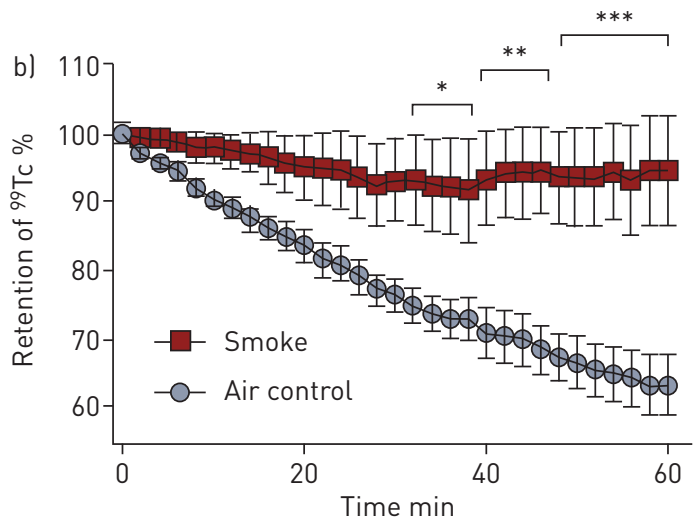

$2 \%$

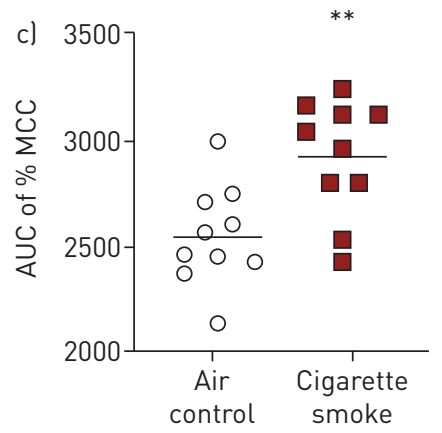

d)

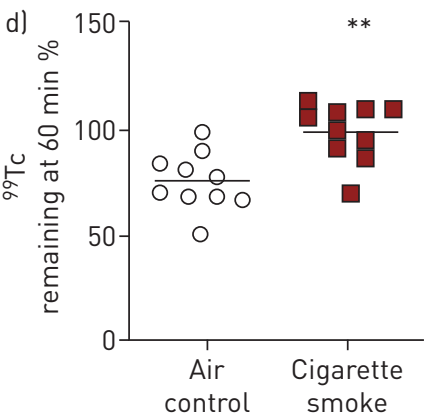

FIGURE 1 In vivo mucociliary clearance (MCC) is impaired in a ferret model of chronic obstructive pulmonary disease. a) Representative images depicting percentage clearance of DTPA-conjugated technetium-99 $\left({ }^{99} \mathrm{Tc}\right)$ from the lungs over time in ferrets exposed to room air or nose-only cigarette smoke for 6 months. Red: areas of maximal clearance $\left(\sim 2 \% \cdot \mathrm{min}^{-1}\right)$; dark blue/black: regions of minimal clearance $\left(0 \% \cdot \mathrm{min}^{-1}\right)$. b) Quantification of percentage clearance over time. c) Area under the curve (AUC) for percentage retention after 60 min. d) Percentage of total Tc-DTPA remaining after 60 min of $\gamma$-imaging. $n=8-12$ animals per group; ${ }^{*}: p<0.05,{ }^{* *}: p<0.01,{ }^{* *}: p<0.001$ compared to air control, using two-way ANOVA.

measured simultaneously and in a co-localised fashion. In preparation for this analysis, we report each individual region of interest replicate in supplementary figure S3 by smoke exposure status. MCT was again diminished by $27.4 \%$ (mean, 95\% CI smoke 7.24\%, 6.34-8.13\% versus control 9.97\%, 8.80-11.14\%) in COPD ferret airways (supplementary figure S3a), and was also associated with a $8.8 \%$ reduction (smoke $5.91 \%, 5.59-6.24$ versus control 6.48\%, 6.01-6.96\%) in ASL depth (supplementary figure S3b) and a $2.9 \%$ reduction (smoke $3.05 \%, 2.97-3.13 \%$ versus control 3.14\%, 3.07-3.21\%) in PCL depth (supplementary figure S3c), while CBF (smoke 10.63\%, 10.37-10.89\% versus control 10.44\%, 10.19-10.68\%) was relatively unchanged (supplementary figure S3d). We then conducted univariate analysis with steady-state (pre-carbachol stimulation) MCT as the dependent variable; by ferret, reduced mean MCT was associated with smoke exposure and mean CBF (supplementary table S1). To evaluate for independent contributors to the MCT defect, we then executed a linear mixed model that controlled for repeated measures within ferrets, but included each of the variables in the univariate analysis, in addition to experimental cohort and each of two measurements made prior to carbachol stimulation, to control for their effect (table 1). Independent predictors of MCT included smoking, mean ASL depth and mean CBF, whereas sex, baseline measurement replicate and experimental cohort were not significant. In aggregate, these data indicate that smoking confers deleterious effects on mucus transport, and airway dehydration and ciliary dysfunction are key contributors to this dysfunction.

\section{Assessment of primary human airway cells}

We confirmed these findings using primary $\mathrm{HBE}$ cells isolated from normal nonsmokers and exposed to cigarette smoke, noting that $\mathrm{HBE}$ cells do not capture the full complexity of the mucociliary transport apparatus as compared to tissues such as secretions from glands. Cigarette smoke exposure reduced ASL depth (supplementary figure S4a and b), PCL depth (supplementary figure S4c), CBF (supplementary figure S4d) and MCT rate (supplementary figure S4e) in vitro using primary HBE cells imaged via $\mu \mathrm{OCT}$, reflecting the deleterious effects of smoking on ion transport-dependent fluid secretion of airway epithelial cells [29]. Overall, our findings in primary human airway epithelial cells and a ferret model of COPD indicate that chronic cigarette smoke diminishes MCT, consistent with prior observations $[12,22,23]$, and does so by perturbing several aspects of the functional microanatomy. 
a)
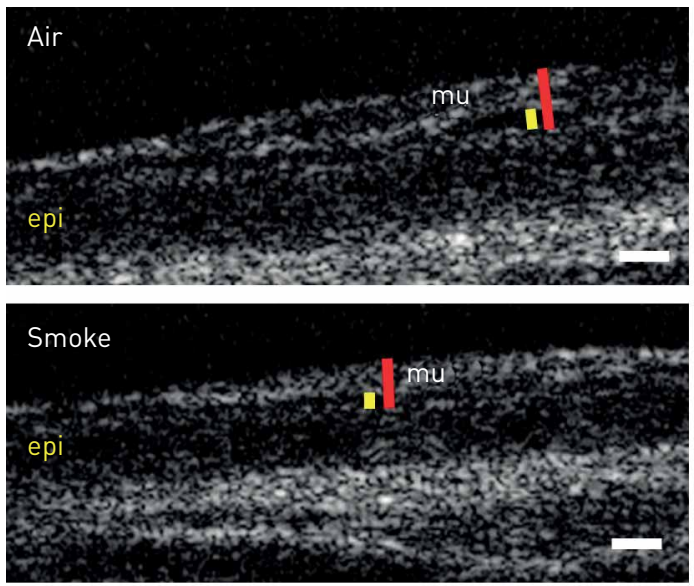

b)
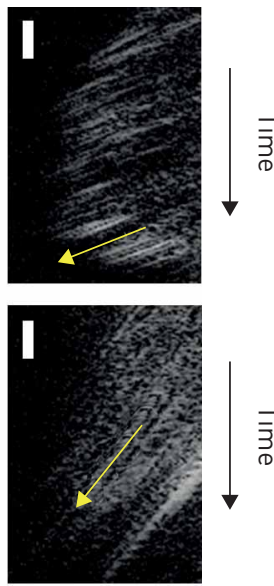


FIGURE 2 A ferret model of chronic obstructive pulmonary disease exhibits abnormal local airway epithelial anatomy and function. a) Representative micro-optical coherence tomography ( $\mu \mathrm{OCT}$ ) stills of trachea excised from air- or smoke-exposed ferrets; b) representative re-slices of $\mu \mathrm{OCT}$ videos for mucociliary transport (MCT) rate quantification; c) MCT rate; d) airway surface liquid (ASL); e) periciliary layer (PCL); and f) ciliary beat frequency (CBF) were quantified for each ferret. $n=27$ air control and $n=28$ smoke-exposed; *: $p<0.05$ compared to air control, as assessed using unpaired Mann-Whitney test. Data are presented as box-and-whisker (median \pm quartiles, using Tukey's method). epi: epithelium; mu: mucus.

The effect of stimulated mucus secretion

Activating glandular secretion through cholinergic stimulus can accelerate mucus transport in normal airways [30, 31], but has important deleterious effects in CF [28, 32, 33], inducing stasis as mucus overwhelms the ion transport apparatus, precipitating adhesive mucus, particularly at the gland duct [32, 33]. Carbachol stimulation of normal ferret trachea increased mucus fluid secretion, as reflected by ASL

TABLE 1 Linear mixed-effects model identifying predictors of steady-state mucociliary transport

\begin{tabular}{|c|c|c|}
\hline & Estimate $\pm s E$ & $p$-value \\
\hline Sex & $-0.7922 \pm 1.1016$ & 0.4768 \\
\hline Smoke & $-3.1912 \pm 0.9658$ & 0.0022 \\
\hline ASL & $-0.6433 \pm 0.2244$ & 0.0072 \\
\hline PCL & $4.0598 \pm 2.2289$ & 0.0778 \\
\hline CBF & $1.2990 \pm 0.4684$ & 0.0085 \\
\hline Baseline replicate & $-0.2009 \pm 0.5696$ & 0.7257 \\
\hline Cohort A & $2.6826 \pm 3.2254$ & 0.4091 \\
\hline Cohort B & $4.0987 \pm 4.1197$ & 0.3272 \\
\hline Cohort C & $2.1199 \pm 3.8897$ & 0.5892 \\
\hline Cohort D & $7.5397 \pm 5.1513$ & 0.1526 \\
\hline Cohort E & $-1.2199 \pm 2.9494$ & 0.6818 \\
\hline Cohort F & $1.8132 \pm 3.1679$ & 0.5711 \\
\hline Cohort G & $4.9033 \pm 3.4162$ & 0.1607 \\
\hline
\end{tabular}

$\mathrm{n}=55$ ferrets. Bold type represents statistical significance. ASL: airway surface liquid; PCL: periciliary layer; CBF: ciliary beat frequency. $R^{2}=0.56, p<0.001$. 

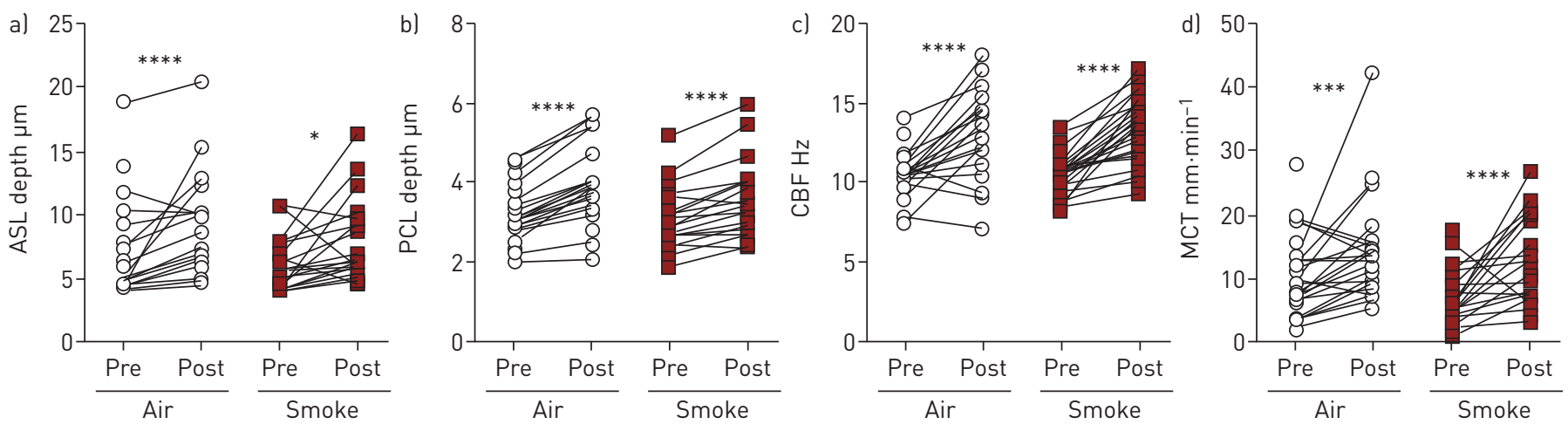

FIGURE 3 Cholinergic stimulation rescues mucus transport in chronic obstructive pulmonary disease ferret trachea. a) Airway surface liquid $(A S L)$; b) periciliary layer (PCL); c) ciliary beat frequency (CBF); and d) mucociliary transport (MCT) were quantified for each animal. Data are plotted as pre- and post-stimulation pairs by individual animal. $\mathrm{n}=27$ air control and $\mathrm{n}=28$ smoke-exposed ferrets; *: $\mathrm{p}<0.05, * * *$ : $\mathrm{p}<0.001$, ****: $p<0.0001$ compared to baseline and based on paired Wilcoxon tests.

and PCL depths $(22.3 \%$ and $20.5 \%$, respectively, $\mathrm{p}<0.0001$; figure 3a and b), stimulated CBF $(25.6 \%$, $\mathrm{p}<0.0001$; figure $3 \mathrm{c})$ and accelerated $\mathrm{MCT}(43.0 \%$, $\mathrm{p}<0.001$; figure $3 \mathrm{~d})$. In contrast to cystic fibrosis $(\mathrm{CF})$ $[28,32,33]$, cholinergic stimulation of ferret trachea following smoke-induced chronic bronchitis had similar effects to that of normal trachea: carbachol significantly increased ASL depth $(24.3 \%, \mathrm{p}<0.05$; figure $3 a)$, PCL depth (13.0\%, $\mathrm{p}<0.0001$; figure 3b), CBF (25.1\%, $\mathrm{p}<0.0001$; figure $3 \mathrm{c}$ ) and MCT $(91.4 \%$, $\mathrm{p}<0.0001$; figure $3 \mathrm{~d}$ ) in smoke-exposed ferrets over baseline. These findings suggest that cholinergic stimulation can partially rescue defects in microanatomical function in smoking-related lung disease, where deficits in $\mathrm{CF}$ transmembrane conductance regulator (CFTR) function are known to occur, but not to the extent observed in CF [12, 22, 34-36].

\section{Mucus viscosity is increased in smoke-exposed ferrets}

To determine whether smoke-exposed ferrets exhibit mucus abnormalities in the airways, as observed in sputum expectorated by COPD patients [14,37], which might contribute to effects not fully explained by airway dehydration, we performed histology on airway tissue from our ferret model, as well as particle tracking microrheology using 500-nm particles in mucus secreted by excised trachea following stimulation (reflecting the contributions of glandular and cellular secretions, rather than mucins adherent to the airway surface). Histologically, ferrets exposed to cigarette smoke exhibited evidence of goblet cell hyperplasia, submucosal gland hypertrophy and overall higher amounts of mucus staining (figure 4a), indicating a chronic bronchitis phenotype consistent with our prior report [16]. Particles in mucus from smoke-exposed ferrets obtained after carbachol stimulation (necessary to capture sufficient volume of nonadherent mucus for analysis) exhibited lower mean-squared displacement over time (figure $4 \mathrm{~b}$ and $c$ ) and thus higher effective viscosity (figure $4 \mathrm{~d}$ ), including a 1.9 -fold increase $(\mathrm{p}<0.05)$ at near static shear stress $(0.6 \mathrm{~Hz})$ (figure $4 \mathrm{e})$. Solid content obtained after stimulation with carbachol demonstrated little difference $(3.0 \pm 0.2 \%$ versus $2.9 \pm 0.2 \%$ control, $\mathrm{p}=0.32)$ (figure $4 \mathrm{f}$ ). While initially surprising, the lack of difference in solid content indicates that the carbachol stimulation of fluid secretion was sufficient to resolve expected differences in solid content, but elevated viscosity nevertheless persisted, probably indicating the irreversibility of changes to mucus with smoke exposure, at least over the short time domains evaluated in these studies.

\section{COPD mucus is more viscous than healthy mucus}

Given that mucus viscosity and solid content have been correlated [32, 38, 39], and elevated viscosity contributes to mucus stasis in CF over and above the effects of airway dehydration alone [28, 33, 40], we next sought to establish whether viscosity played a role in human samples. We performed histology on the same tissues utilised for HBE assays, to survey differences in general appearance of the epithelium and mucus-producing structures across donor groups. Histologically, COPD and healthy smoker surface epithelia exhibited goblet cell hyperplasia and mucus impaction, including the gland ducts, whereas both were minimal in nonsmoker tissue (figure 5). In addition, COPD tissue exhibited submucosal gland hypertrophy, and both healthy smoker and COPD glands stained more intensely with Alcian blue/periodic acid-Schiff compared to nonsmoker samples. Together, this suggests that mucus is more prevalent and intensely expressed as pathology progresses to COPD.

We analysed viscosity of mucus derived from primary HBE cells (supplementary table S2), without pooling secretions from multiple donors, exogenous exposure to cigarette smoke, or cholinergic 
a)
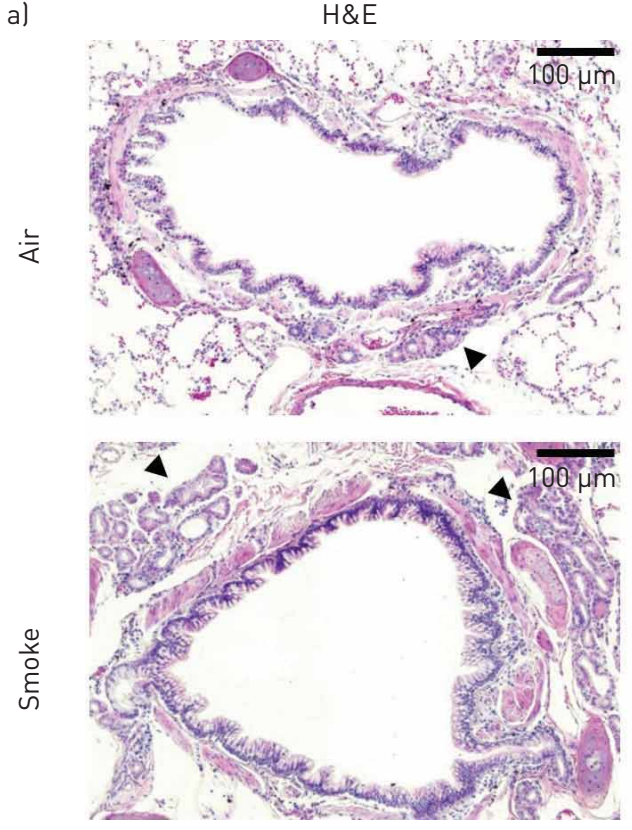
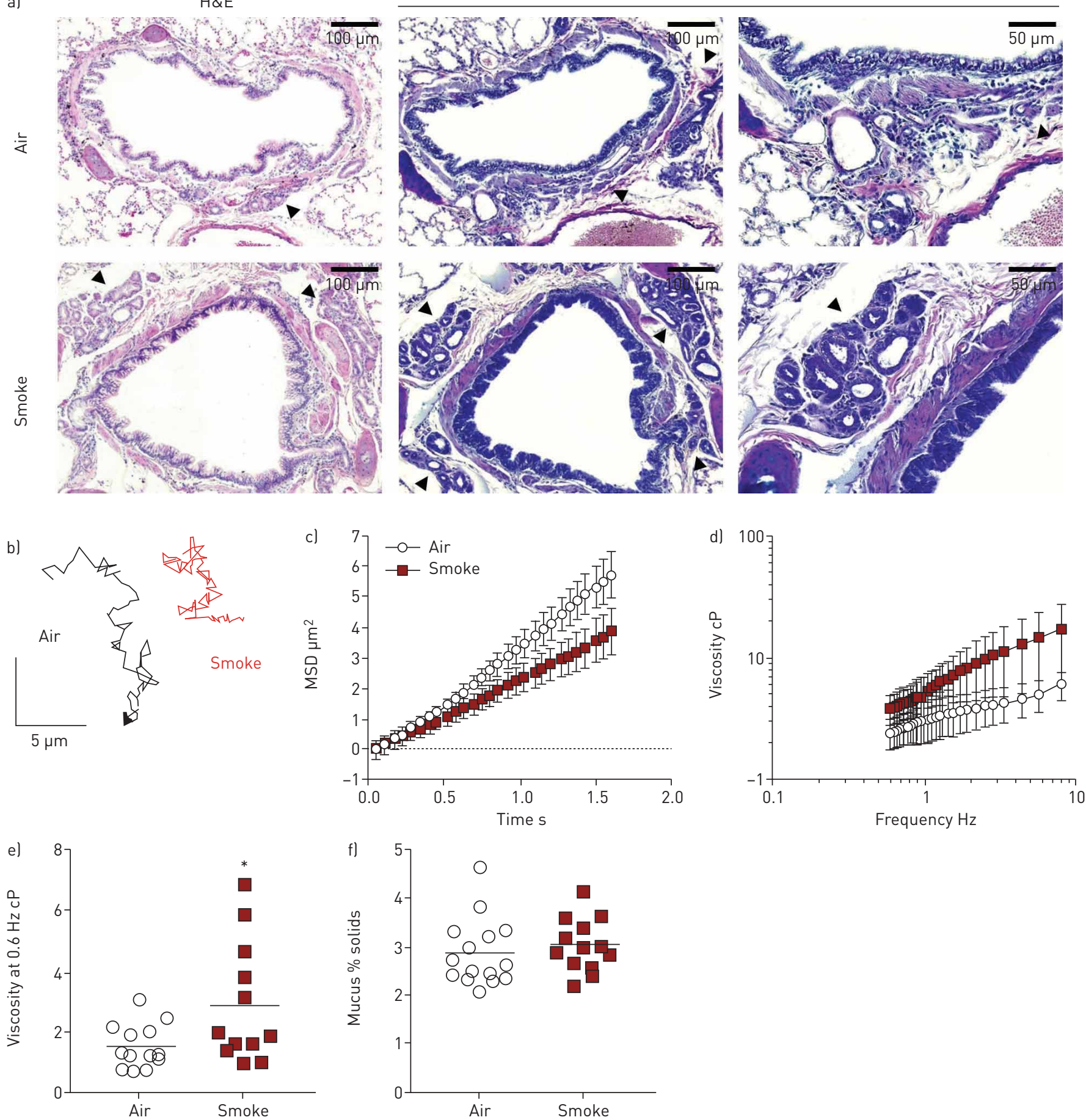

FIGURE 4 Smoke-exposed ferrets exhibit a trend toward increased mucus viscosity. a) Representative haematoxylin and eosin (H\&E) and Alcian blue/periodic acid-Schiff (AB/PAS) staining of tracheal sections and lung tissue from air control and smoke-exposed ferrets. Arrowheads depict submucosal glands. b-e) Particle tracking microrheology was used to measure ferret tracheal mucus viscosity. b) Representative tracings of the Brownian motion of individual 500-nm particles moving through mucus collected from air- or smoke-exposed ferrets; cl mean-squared displacement (MSD) and d) corresponding effective viscosities over a range of frequencies and e) at $0.6 \mathrm{~Hz}$ for each group; f) mucus percentage solid content was calculated from measured wet and dry weights. $n=12-15$ animals per group; *: $p<0.05$ compared to air control, using unpaired t-test.

stimulation, by particle tracking microrheology (figure 6a), noting that this does not include the contribution of gland mucus as the ferret samples do. Mean-squared displacement of 1- $\mu \mathrm{m}$ particles (figure 6b) was markedly reduced in COPD mucus compared to normal and healthy smoker donors 

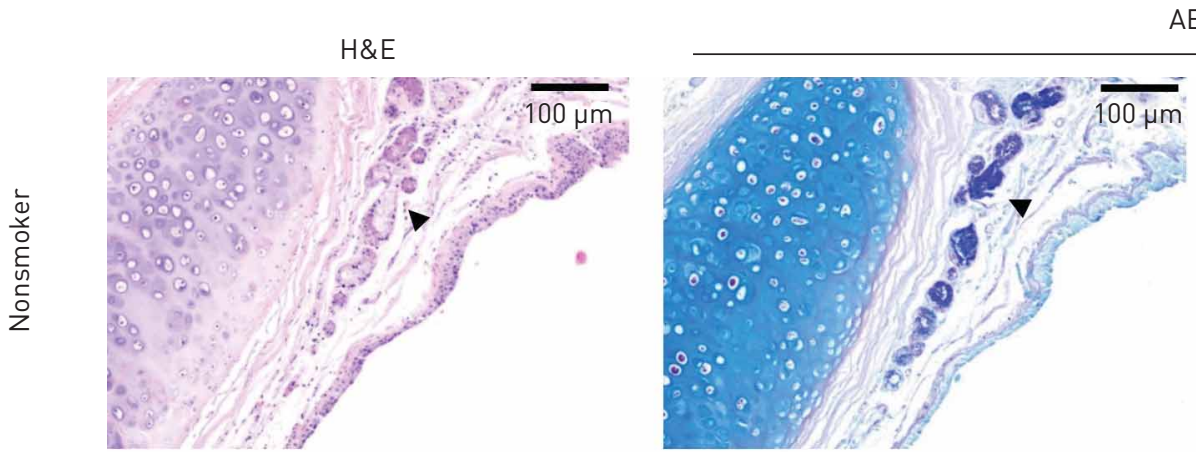

AB/PAS
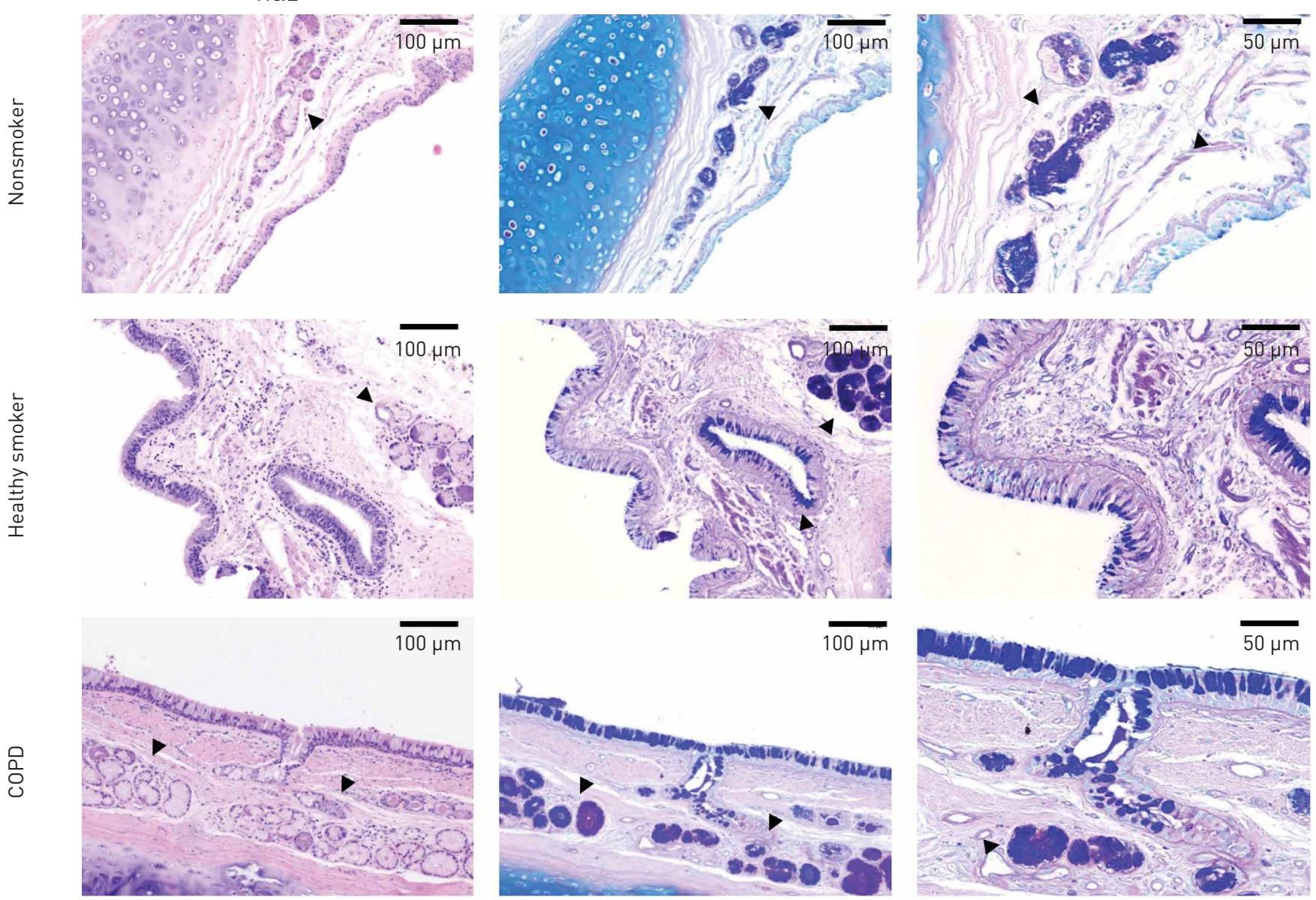

FIGURE 5 Histopathology of normal control, healthy smoker and chronic obstructive pulmonary disease (COPD) human airway tissue. Representative haematoxylin and eosin (H\&E) and Alcian blue/periodic acid-Schiff (AB/PAS) staining of bronchial sections obtained from nonsmoker, healthy smoker and COPD donors. Arrowheads indicate submucosal glands.

$(\mathrm{p}<0.05$ ), corresponding to significantly higher effective viscosity (figure $6 c, p<0.05$ and $\mathrm{p}<0.01$, respectively). At $0.6 \mathrm{~Hz}$ (figure $6 \mathrm{~d}$ ), mucus from COPD donors exhibited viscosity 136 -fold $(\mathrm{p}<0.01$ ) and 59 -fold $(\mathrm{p}<0.05)$ higher than mucus from normal and healthy smokers, respectively. In addition, COPD mucus contained significantly higher percentage solids $(2.77 \pm 0.15 \%)$ compared to nonsmoking controls $(1.57 \pm 0.05 \%, \mathrm{p}<0.0001)$ and nondiseased smokers $(2.01 \pm 0.05 \%, \mathrm{p}<0.0001)$ (figure $6 \mathrm{e}$ ); findings that parallel patient sputum observations [39, 41, 42]. Healthy smoker mucus solid content was significantly higher than that of nonsmoking controls $(\mathrm{p}<0.05)$, reflecting the intermediate phenotype of nondiseased smokers. Noting the absent contribution of glands in this model, we observed that mucus solid content and effective viscosity were positively correlated $\left(\mathrm{R}^{2}=0.7652, \mathrm{p}=0.01\right)$ (figure $6 \mathrm{f}$ ).

\section{Discussion}

While cigarette smoke exposure has been associated with reduced MCT and other defects of mucus in vitro $[11,12,22,23]$, this has never been shown in a relevant animal model, and the specific biophysical mechanisms by which smoke contributes to airway epithelial dysfunction and subsequent mucus obstruction in COPD pathogenesis remain unclear. Here, we demonstrate that cigarette smoke impairs the airway functional microanatomy in a novel COPD ferret model [16], and support findings by several complementary model systems, from in vitro analysis to unique assessments following in vivo cigarette smoke exposure. We show that diminished mucus transport is a major consequence of smoke exposure, and is impacted by airway dehydration, ciliary beating and mucus viscosity. We further show that cholinergic stimulation of the airway epithelium can partially, but not completely rescue these transport defects by augmenting fluid secretion, distinct from CF, in which it can worsen mucus stasis [33]. These 
a)
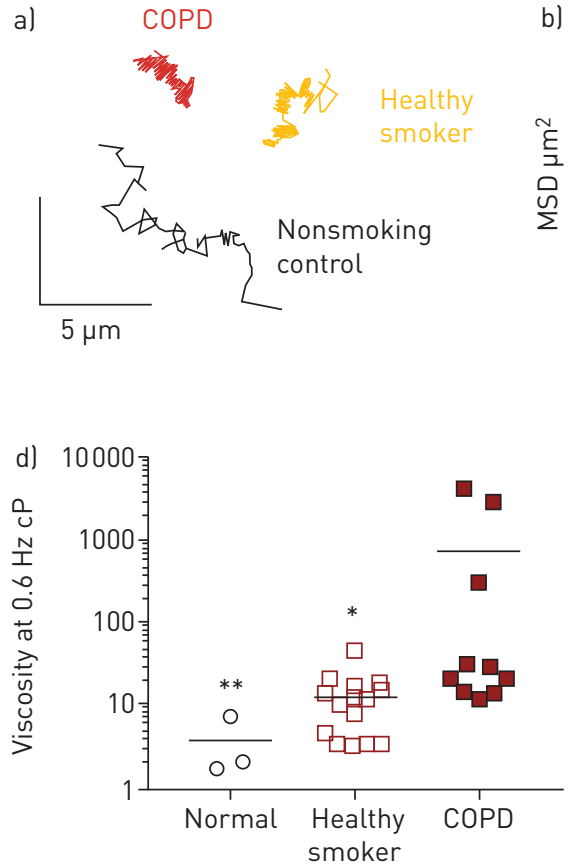
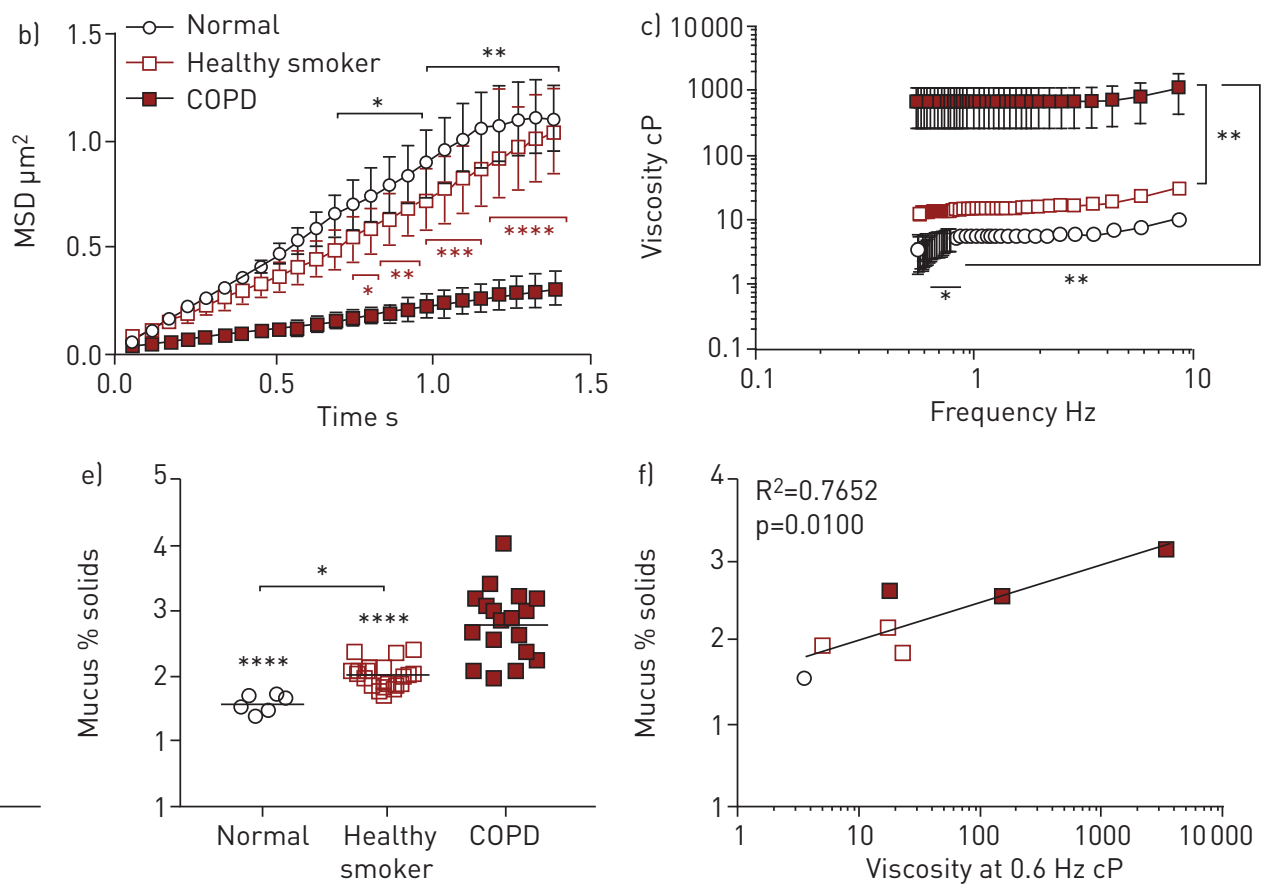

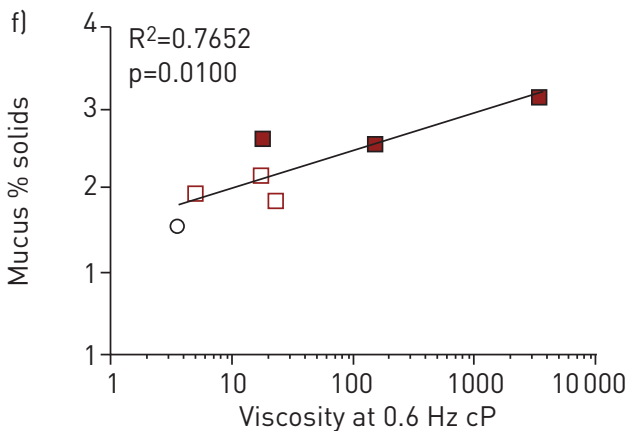

FIGURE 6 Healthy smoker and chronic obstructive pulmonary disease (COPD) human bronchial epithelial (HBE) cells perpetuate mucus abnormalities. a) Representative tracings of the Brownian motion of individual 1- $\mu$ m particles moving through normal control, healthy smoker and COPD mucus. Particle tracking microrheology (PTM) was used to measure b) mean-squared displacement (MSD) of particles over time, within mucus secreted by HBE cells (normal, healthy smoker, COPD), from which c) effective viscosity was calculated; d) comparison of effective viscosity of each group at $0.6 \mathrm{~Hz}$; e) mucus percentage solids content by weight was calculated for each donor group; ff the relationship between mucus solid content and effective viscosity for each donor. $n=1$ normal, $n=3$ healthy smoker, $n=3$ COPD donors (with two to four samples per donor for PTM, and six samples per donor for percentage solids); *: $p<0.05,{ }^{* *}$ : $p<0.01,{ }^{* * * *} p<0.0001$ compared to b) COPD using two-way ANOVA c) healthy smoker unless otherwise denoted with two-way ANOVA or d,e) COPD unless otherwise denoted by one-way ANOVA.

findings implicate both airway epithelial dysfunction and mucus viscoelasticity as potential therapeutic targets for chronic bronchitis and other muco-obstructive diseases.

Our $\mu$ OCT data confirm that apical cigarette smoke exposure impairs ASL and MCT in vitro [11, 12, 22, 23]. However, HBE cells do not fully replicate the airway surface, which contains submucosal glands. To examine this more comprehensively than is possible in cell monolayers, we employed an experimental in vivo model that could capture contributions of both the surface epithelium and airway glands. To this end, ferrets more closely resemble humans in terms of airway goblet cell and glandular distribution [17], and are large enough to provide an accurate representation of mucus properties. In vivo MCC assays demonstrated higher retention of Tc-DTPA in smoke-exposed animals. While human studies largely rely on radiographic clearance of Tc-sulfur colloid, minimal clearance occurred in ferrets due to excessive alveolar deposition, whereas Tc-DTPA nebulisation distributed to the airways in sufficient quantity. Supporting our approach, multiple reports [24, 43,44] confirm the utility of DTPA particles in distinguishing absorptive clearance from MCC if absorption can be independently estimated and accounted for, as we have done here. In addition, it has been reported that DTPA clearance by absorption is only $\sim 50 \%$ of its total clearance [44], suggesting sufficient discrimination for estimating MCC with appropriate normalisation. Inflammation reduces epithelial integrity, causing increased absorption of inhaled particles [45]. Thus, our observation of greater absorption of Tc-DTPA in smoke-exposed ferret lungs when compared to control ferret lungs is consistent with what may be expected in COPD lungs with exaggerated inflammation. We find it important to note that despite increased absorptive clearance of Tc-DTPA in smoke-exposed ferrets, total amount of Tc-DTPA remaining in lungs at the end of $60 \mathrm{~min}$ was higher than that of controls, suggesting significantly impaired MCC. KARACAVUS and INTEPE [46] have reported that differences in MCC as a factor of current smoking status can be distinguished in control and asthma subjects using Tc-DTPA, adding further support to our approach.

The defect in MCC translated to impaired tracheal MCT, indicating that mucus clearance is delayed in both the upper and lower airways of our model, and that the airway surface microenvironment is important in determining mucus transport, probably due to the co-interaction of functional parameters. Although the effect of cigarette smoke was much more dramatic in MCC measurements in vivo compared 
to tracheal MCT assessments, it should be noted that MCC estimates take into account the entire tracheobronchial tree (including smaller airways, in which mucus obstruction may occur earlier and more dramatically, or which may drop-out entirely) [47], whereas ex vivo tracheal MCT only provides us measures in the most ciliated portion of the airways (which also contains the highest glandular and goblet cell contributions). Additionally, it is possible that cigarette smoke exposure in this model affects the whole lung more than the trachea itself, and may be dependent on dwell time in the respective regions, as well as deposition of particulate matter of various sizes. While our MCC method accounted for absorptive clearance that also occurs with DTPA, it is possible residual differences in absorption for which our calculations did not account.

Given the power of $\mu \mathrm{OCT}$ to assess covariates of the airway microanatomy simultaneously and in a co-localised manner, we used this technique to measure smoke-induced changes in excised ferret trachea, and to examine their independent contributions, controlled for multiple replicates. Specifically, we used $\mu \mathrm{OCT}$-acquired measures to identify how individual functional parameters interact with MCT, and which are important in predicting mucus transport. As observed in other smoke exposure models, we found significant reductions in MCT (corroborating our in vivo MCC results) and CBF in smoke-exposed ferrets. When accounting for multiple contributors to delayed mucus clearance, mixed model analysis revealed that augmented ASL (airway hydration) and CBF (ciliary function) had positive effects on steady-state MCT, while smoke exposure had a negative impact. It should be noted that there could be other factors not easily measured in our model that may impact mucus clearance at all levels, such as ASL pH, DNA content, mucus adherence to the epithelial surface, differential presence of specific mucins and mucin cross-linking; these deserve further study to examine their potential. Increased mucin secretion and concentration have been suggested as another mechanism leading to thicker mucus $[14,39]$, were borne out by pathologic analysis of ferret tissues and the bronchi of human cell donors.

Based on the fact that MCC and MCT were clearly diminished by smoking, but deficits in CBF and airway hydration did not explain the entirety of the defect, we used microrheological methods to assess the viscosity of normal (non-CF, non-COPD) and COPD mucus from cell monolayers and ferret tissues, since this could account for additional deficits in MCT. Similar to observations in patient sputum [37, 39], mucus collected from COPD HBE cells exhibited higher viscosity than that of nondiseased cells, even without additional cigarette smoke exposure, indicating an intrinsic defect in COPD mucus. To the best of our knowledge, this is the first evidence of increased viscosity in mucus secreted by an in vitro COPD model that rules out the presence of covariates unrelated to the epithelium itself, and was confirmed in samples from well-controlled ferret exposures. Additionally, COPD HBE mucus demonstrated higher percentage solids by weight, which is in agreement with recent studies in normal and COPD cells [39] and sputum [14, 41]; although differences between groups were small, HBE cells lack submucosal glands, and are probably missing a significant contribution present in vivo. These data suggest exposure-related alterations in mucin structure or the overall mucus meshwork, potentially fruitful areas for additional research targeting correction of abnormal mucus.

Interestingly, mucus secreted by cells from healthy smokers yielded solid content and viscosity at levels intermediary to nonsmoker and COPD samples, as well as evidence of goblet cell hyperplasia and increased epithelial mucus staining. While limited by small numbers due to the availability of clinically characterised transplant specimens, this suggests that airways exposed to cigarette smoke not only produce more mucus, but it is more viscous, even without (or prior to) disease being established, and that continual and chronic exposure to high levels of inhaled irritants may increase mucus production and impair mucus clearance even in a nonpathological state. This, in addition to higher mucin concentrations observed in sputum produced by nondiseased symptomatic smokers compared to asymptomatic ones [14], may provide a significant mechanistic basis underlying mucus obstruction in COPD and could prove useful for diagnostic purposes, or even a potential prevention target should smoking cessation prove unsuccessful. This may also explain epidemiological associations of respiratory infections with smoking [48].

Unlike observations in CF airways following cholinergic stimulation of secretion [30, 32, 33], we demonstrated that cholinergic stimulus, applied from the serosal surface then allowed to equilibrate, rescued MCT defects in COPD ferret tracheae even though decrements in ASL and PCL persisted. This suggests that COPD airway epithelia have the capacity to overcome this impairment, potentially because smoke-exposed epithelia still express functional anion channels, including present but diminished CFTR, which cholinergic-stimulated calcium ion release may potentially activate [49, 50]. This benefit may precede complete rehydration of airway mucus. Based on this, activating ion transport to normalise epithelial secretion or hydration represents a viable therapeutic avenue, as previously proposed to address MCT defects resulting from smoke-induced dehydration [12, 22, 23]. Anticholinergic therapies, i.e. long-acting muscarinic antagonists (LAMAs), reduce cholinergic tone to improve airway conductance, and can reduce mucus production when used in conjunction with long-acting $\beta$-adrenoceptor agonists 
(LABAs), which may enhance ciliary beating and thus MCC, they can lower exacerbation frequency and severity [51]. Since LAMAs are antimuscarinic agents, we speculate that compounds targeting the nicotinic arm of cholinergic pathways may also be useful, particularly since pathway disruption downstream of smoke exposure includes nicotinic receptors, and would be expected to confer distinct effects on epithelial function from that of the smooth muscle [52-54]. Given that carbachol, a nonspecific cholinergic that can bind muscarinic and nicotinic receptors, increased fluid secretion as well as MCT in ferrets and in a synergistic fashion with cAMP agonists [55], this suggests that the nicotinic pathway, when targeted selectively, may be a viable and novel target to improve mucus clearance without interfering with the standard use of antimuscarinic agents to treat COPD. Future studies to assess the effects of independent activation of nicotinic and muscarinic receptors would be necessary to elucidate whether this approach is feasible without compromising the beneficial effects of anticholinergic therapy on smooth muscle tone and mucus production in these patients, much like the complementary use of LABAs. Because our findings support augmenting ion channel secretion to attenuate mucus clearance defects, activation or potentiation of CFTR itself represents an alternative approach that is currently under investigation [22, 23].

In summary, we demonstrate using several complementary models that chronic cigarette smoke exposure reduces mucociliary transport by impairing airway epithelial function (ciliary dysfunction, reduced hydration), and by altering mucus properties (increasing solid content and viscosity). Given these findings, augmenting aspects of the airway functional microanatomy to facilitate mucus transport in chronic bronchitis may prove beneficial for improving overall mucus clearance. Due to the heterogeneity of COPD, it may be necessary to combine therapies targeting specific mechanisms underlying its pathogenesis on an individual basis to ultimately slow disease progression.

Acknowledgements: The authors acknowledge Ariane Fulce, Jana Frost-Deleersnyder, and the Tissue Collection and Banking Facility at the University of Alabama at Birmingham (UAB; Birmingham, AL, USA) for services related to airway tissue procurement and histopathology; Sharon Samuel and the UAB Small Animal Imaging Shared Facility for their assistance with mucociliary clearance imaging; Ken Chu and Hui Minh Leung (Wellman Center for Photomedicine, Massachusetts General Hospital, Boston, MA, USA) for their maintenance of the micro-optical coherence tomography $(\mu \mathrm{OCT})$ system and expertise in developing and updating custom MatLab scripts for $\mu$ OCT image analysis; and Greg Duncan and Siddharth Shenoy (Johns Hopkins University, Bethesda, MD, USA) for their assistance with fluorescent particles used for particle tracking microrheology. The authors also thank Heather Hathorne (UAB, Birmingham, AL, USA) for regulatory support regarding work with human subjects and the patients who donated their organs for these experiments.

Author contributions: V.Y. Lin performed rheologic measurement of mucus samples, and analysed the data. V.Y. Lin and S.E. Birket optimised the tracheal explant imaging protocol, performed $\mu \mathrm{OCT}$ imaging of airway samples, and analysed the data. S.V. Raju and H. Kim performed in vivo MCC assays and analysed the resulting images. S.A. Byzek performed in vivo smoke exposures. S.V. Raju, S.A. Byzek and N. Kaza performed animal necropsies for trachea studies. L.J. Edwards performed statistical modelling of $\mu \mathrm{OCT}$ data. J. LaFontaine performed histologic analysis of bronchial and lung tissue. S.A. Byzek measured TPM output throughout exposure studies. M. Mazur harvested and grew cells from human donor tissue for primary cell culture. J. Hanes provided fluorescent nanoparticles and expertise for particle tracking experiments. G.J. Tearney developed $\mu \mathrm{OCT}$ technology and all related analytical methods, and provided technical support and expertise. V.Y. Lin, S.E. Birket, S.V. Raju and S.M. Rowe contributed to experimental design and data interpretation. V.Y. Lin and S.M. Rowe wrote the manuscript, which was reviewed by N. Kaza, S.E. Birket, S.V. Raju, H. Kim, M. Mazur, S.A. Byzek, J. Hanes and G.J. Tearney.

Support statement: This study was financially supported by the National Institutes of Health (NIH grants P30DK072482, R35HL135816 and F31HL134225), and the Flight Attendant Medical Research Institute. Funding information for this article has been deposited with the Crossref Funder Registry.

Conflict of interest: V.Y. Lin has nothing to disclose. N. Kaza has nothing to disclose. S.E. Birket has nothing to disclose. H. Kim has nothing to disclose. L.J. Edwards has nothing to disclose. J. LaFontaine has nothing to disclose. L. Liu has a patent "Method for functional investigation of respiratory airways and other ciliated tissues using $\mu$ OCT" pending. M. Mazur has nothing to disclose. S.A. Byzek has nothing to disclose. J. Hanes is founder and owner of company stock (which is subject to certain rules and restrictions under Johns Hopkins University policy) of GrayBug Vision, Inc., and Kala Pharmaceuticals, Inc., outside the submitted work. G.J. Tearney has patents 14/240,938 and 12826303.5 pending. S. V. Raju has nothing to disclose. S.M. Rowe reports grants from Bayer, Forest Research Institute, AstraZeneca, N30/ Nivalis, Novartis, Galapagos/AbbVie, Proteostasis, PTC Therapeutics and Eloxx, grants and personal fees for consultancy from Celtaxsys, personal fees for consultancy and advisory board work and in kind support for clinical trial work from Vertex Pharmaceuticals Incorporated, personal fees for consultancy from Bayer and Novartis, outside the submitted work; and has a patent "Use of OCT as a diagnostic modality for diseases of mucus clearance" issued.

\section{References}

1 Global, regional, and national life expectancy, all-cause mortality, and cause-specific mortality for 249 causes of death, 1980-2015: a systematic analysis for the Global Burden of Disease Study 2015. Lancet 2016; 388: 1459-1544.

2 Mannino DM, Buist AS. Global burden of COPD: risk factors, prevalence, and future trends. Lancet 2007; 370: 765-773. 
3 Mannino DM, Gagnon RC, Petty TL, et al. Obstructive lung disease and low lung function in adults in the United States: data from the National Health and Nutrition Examination Survey, 1988-1994. Arch Intern Med 2000; 160: 1683-1689.

4 Schikowski T, Mills IC, Anderson HR, et al. Ambient air pollution: a cause of COPD? Eur Respir J 2014; 43: 250-263.

5 Salvi SS, Barnes PJ. Chronic obstructive pulmonary disease in non-smokers. Lancet 2009; 374: $733-743$.

6 Saetta M, Turato G, Baraldo S, et al. Goblet cell hyperplasia and epithelial inflammation in peripheral airways of smokers with both symptoms of chronic bronchitis and chronic airflow limitation. Am J Respir Crit Care Med 2000; 161: 1016-1021.

7 Morgan L, Pearson M, de Iongh R, et al. Scintigraphic measurement of tracheal mucus velocity in vivo. Eur Respir J 2004; 23: 518-522.

8 Möller W, Felten K, Sommerer K, et al. Deposition, retention, and translocation of ultrafine particles from the central airways and lung periphery. Am I Respir Crit Care Med 2008; 177: 426-432.

9 Haswell LE, Hewitt K, Thorne D, et al. Cigarette smoke total particulate matter increases mucous secreting cell numbers in vitro: a potential model of goblet cell hyperplasia. Toxicol In Vitro 2010; 24: 981-987.

10 Innes AL, Woodruff PG, Ferrando RE, et al. Epithelial mucin stores are increased in the large airways of smokers with airflow obstruction. Chest 2006; 130: 1102-1108.

11 Clunes LA, Davies CM, Coakley RD, et al. Cigarette smoke exposure induces CFTR internalization and insolubility, leading to airway surface liquid dehydration. FASEB J 2012; 26: 533-545.

12 Rasmussen JE, Sheridan JT, Polk W, et al. Cigarette smoke-induced $\mathrm{Ca}^{2+}$ release leads to cystic fibrosis transmembrane conductance regulator (CFTR) dysfunction. J Biol Chem 2014; 289: 7671-7681.

13 Chen EY, Sun A, Chen CS, et al. Nicotine alters mucin rheological properties. Am J Physiol Lung Cell Mol Physiol 2014; 307: L149-L157.

14 Kesimer M, Ford AA, Ceppe A, et al. Airway mucin concentration as a marker of chronic bronchitis. $N$ Engl $J$ Med 2017; 377: 911-922.

15 Livraghi-Butrico A, Grubb BR, Wilkinson KJ, et al. Contribution of mucus concentration and secreted mucins Muc5ac and Muc5b to the pathogenesis of muco-obstructive lung disease. Mucosal Immunol 2017; 10 : 395-407.

16 Raju SV, Kim H, Byzek SA, et al. A ferret model of COPD-related chronic bronchitis. JCI Insight 2016; 1: e87536

17 Hajighasemi-Ossareh M, Borthwell RM, Lachowicz-Scroggins M, et al. Distribution and size of mucous glands in the ferret tracheobronchial tree. Anat Rec 2013; 296: 1768-1774.

18 Sun X, Olivier AK, Liang B, et al. Lung phenotype of juvenile and adult cystic fibrosis transmembrane conductance regulator-knockout ferrets. Am J Respir Cell Mol Biol 2014; 50: 502-512.

19 Groneberg DA, Chung KF. Models of chronic obstructive pulmonary disease. Respir Res 2004; 5: 18.

20 Wright JL, Cosio M, Churg A. Animal models of chronic obstructive pulmonary disease. Am J Physiol Lung Cell Mol Physiol 2008; 295: L1-L15.

21 Liu L, Chu KK, Houser GH, et al. Method for quantitative study of airway functional microanatomy using micro-optical coherence tomography. PLoS One 2013; 8: e54473.

22 Sloane PA, Shastry S, Wilhelm A, et al. A pharmacologic approach to acquired cystic fibrosis transmembrane conductance regulator dysfunction in smoking related lung disease. PLoS One 2012; 7: e39809.

23 Raju SV, Lin VY, Liu L, et al. The cystic fibrosis transmembrane conductance regulator potentiator ivacaftor augments mucociliary clearance abrogating cystic fibrosis transmembrane conductance regulator inhibition by cigarette smoke. Am J Respir Cell Mol Biol 2017; 56: 99-108.

24 Corcoran TE, Thomas KM, Brown S, et al. Liquid hyper-absorption as a cause of increased DTPA clearance in the cystic fibrosis airway. EJNMMI Res 2013; 3: 14

25 Cho HJ, Joo NS, Wine JJ. Mucus secretion from individual submucosal glands of the ferret trachea. Am J Physiol Lung Cell Mol Physiol 2010; 299: L124-L136.

26 Chu KK, Mojahed D, Fernandez CM, et al. Particle-tracking microrheology using micro-optical coherence tomography. Biophys J 2016; 111: 1053-1063.

27 Liu L, Shastry S, Byan-Parker S, et al. An autoregulatory mechanism governing mucociliary transport is sensitive to mucus load. Am J Respir Cell Mol Biol 2014; 51: 485-493.

28 Birket SE, Chu KK, Liu L, et al. A functional anatomic defect of the cystic fibrosis airway. Am J Respir Crit Care Med 2014; 190: 421-432.

29 Rab A, Rowe SM, Raju SV, et al. Cigarette smoke and CFTR: implications in the pathogenesis of COPD. Am Physiol Lung Cell Mol Physiol 2013; 305: L530-L541.

30 Ermund A, Meiss LN, Dolan B, et al. The mucus bundles responsible for airway cleaning are retained in cystic fibrosis and by cholinergic stimulation. Eur Respir J 2018; 52: 1800457.

31 Jeong JH, Joo NS, Hwang PH, et al. Mucociliary clearance and submucosal gland secretion in the ex vivo ferret trachea. Am J Physiol Lung Cell Mol Physiol 2014; 307: L83-L93.

32 Birket SE, Davis JM, Fernandez CM, et al. Development of an airway mucus defect in the cystic fibrosis rat. JCI Insight 2018; 3: 97199.

33 Hoegger MJ, Fischer AJ, McMenimen JD, et al. Impaired mucus detachment disrupts mucociliary transport in a piglet model of cystic fibrosis. Science 2014; 345: 818-822.

34 Cantin AM, Hanrahan JW, Bilodeau G, et al. Cystic fibrosis transmembrane conductance regulator function is suppressed in cigarette smokers. Am J Respir Crit Care Med 2006; 173: 1139-1144

35 Raju SV, Jackson PL, Courville CA, et al. Cigarette smoke induces systemic defects in cystic fibrosis transmembrane conductance regulator function. Am J Respir Crit Care Med 2013; 188: 1321-1330.

36 Courville CA, Tidwell S, Liu B, et al. Acquired defects in CFTR-dependent $\beta$-adrenergic sweat secretion in chronic obstructive pulmonary disease. Respir Res 2014; 15: 25

37 Serisier DJ, Carroll MP, Shute JK, et al. Macrorheology of cystic fibrosis, chronic obstructive pulmonary disease \& normal sputum. Respir Res 2009; 10: 63.

38 Ma JT, Tang C, Kang L, et al. Cystic fibrosis sputum rheology correlates with both acute and longitudinal changes in lung function. Chest 2018; 154: 370-377.

39 Hill DB, Vasquez PA, Mellnik J, et al. A biophysical basis for mucus solids concentration as a candidate biomarker for airways disease. PLoS One 2014; 9: e87681. 

mucin secretion and link cystic fibrosis with its mucus phenotype. J Exp Med 2012; 209: 1263-1272.

41 Anderson WH, Coakley RD, Button B, et al. The relationship of mucus concentration (hydration) to mucus osmotic pressure and transport in chronic bronchitis. Am J Respir Crit Care Med 2015; 192: 182-190.

42 Lin VY, Fain MD, Jackson PL, et al. Vaporized e-cigarette liquids induce ion transport dysfunction in airway epithelia. Am J Respir Cell Mol Biol 2019; 61: 162-173.

43 Dawkins L, Corcoran O. Acute electronic cigarette use: nicotine delivery and subjective effects in regular users. Psychopharmacology 2014; 231: 401-407.

44 Locke LW, Myerburg MM, Markovetz MR, et al. Quantitative imaging of airway liquid absorption in cystic fibrosis. Eur Respir J 2014; 44: 675-684.

45 Sundram FX. Clinical studies of alveolar-capillary permeability using technetium-99m DTPA aerosol. Ann Nucl Med 1995; 9: 171-178.

46 Karacavus S, Intepe YS. The role of Tc-99m DTPA aerosol scintigraphy in the differential diagnosis of COPD and asthma. Clin Respir J 2015; 9: 189-195.

47 McDonough JE, Yuan R, Suzuki M, et al. Small-airway obstruction and emphysema in chronic obstructive pulmonary disease. N Engl J Med 2011; 365: 1567-1575.

48 Bagaitkar J, Demuth DR, Scott DA. Tobacco use increases susceptibility to bacterial infection. Tob Induc Dis 2008; 4: 12 .

49 Gustafsson JK, Lindén SK, Alwan AH, et al. Carbachol-induced colonic mucus formation requires transport via NKCC1, K ${ }^{+}$channels and CFTR. Pflugers Arch 2015; 467: 1403-1415.

50 Thiagarajah JR, Song Y, Haggie PM, et al. A small molecule CFTR inhibitor produces cystic fibrosis-like submucosal gland fluid secretions in normal airways. FASEB J 2004; 18: 875-877.

51 Beeh KM, Burgel PR, Franssen FME, et al. How do dual long-acting bronchodilators prevent exacerbations of chronic obstructive pulmonary disease? Am J Respir Crit Care Med 2017; 196: 139-149.

52 Maouche K, Medjber K, Zahm JM, et al. Contribution of $\alpha 7$ nicotinic receptor to airway epithelium dysfunction under nicotine exposure. Proc Natl Acad Sci USA 2013; 110: 4099-4104.

53 Rosas-Ballina M, Goldstein RS, Gallowitsch-Puerta M, et al. The selective $\alpha 7$ agonist GTS-21 attenuates cytokine production in human whole blood and human monocytes activated by ligands for TLR2, TLR3, TLR4, TLR9, and RAGE. Mol Med 2009; 15: 195-202.

54 Galle-Treger L, Suzuki Y, Patel N, et al. Nicotinic acetylcholine receptor agonist attenuates ILC2-dependent airway hyperreactivity. Nat Commun 2016; 7: 13202.

55 Joo NS, Jeong JH, Cho HJ, et al. Marked increases in mucociliary clearance produced by synergistic secretory agonists or inhibition of the epithelial sodium channel. Sci Rep 2016; 6: 36806 